\title{
MATERIAL DISTRIBUTION DESIGN FOR A PIEZOELECTRIC ENERGY HARVESTER DISPLAYING GEOMETRIC NONLINEARITY

\author{
M.A. Philippine ${ }^{i *}$, C.-F. Chiang, J. Salvia, C.M. Jha, S. Yoneoka, T.W. Kenny
} \\ ${ }^{1}$ Stanford University, Stanford, California, USA
}

\begin{abstract}
The optimal use of the piezoelectric for a cantilevered vibrational piezoelectric energy harvester is achieved if the stress is uniform, which can be approached by departing from a simple rectangular-shaped beam. A material distribution algorithm (BESO) is implemented in ANSYS to obtain designs providing a $20 \%$ theoretical increase in power output while maintaining the same maximum stress and less than $5 \%$ change in resonant frequency compared to a rectangular beam. Geometric nonlinearities are included in the finite element model of the MEMS-based energy scavenger. This need is backed by experimental observations of spring hardening. The importance of including nonlinearities in the model is reinforced by the obtention of different structures when BESO is applied with the linear and nonlinear models. The focus of this paper is on the energy harvester design, but there are applications of this method to many MEMS designs.
\end{abstract}

\section{INTRODUCTION}

Simple mechanical structures are commonly employed by MEMS designers; however, these shapes often perform suboptimally. For example, rectangular suspensions [1] and folded beam springs [2] allow for simple device design but may suffer from stress or current concentrations which can limit performance. In this paper, a computational material distribution algorithm is used for the mechanical design of a piezoelectric vibrational energy harvester.

As will be shown in the second section of the theory, this cantilevered device has higher power output levels when the stress in the piezoelectric layer is uniformly distributed. This can be achieved by redesigning the in-plane beam topology using the BESO (Bidirectional Evolutionary Structural Optimization) material distribution algorithm. The algorithm depends on the finite element modeling of the structure, which can take into account the device's stress stiffening behavior.

\section{THEORY}

\section{Harvester Power Output}

The piezoelectric energy scavengers shown in Fig. 1 are intended to respond to vibrations of a car engine near $100 \mathrm{~Hz}$. The power output of a piezoelectric vibrational harvester with a resistive load is given by the following expression [3]:

$$
P=\frac{R C_{b}^{2}}{2 k_{2}^{2} \omega^{2}} \frac{\left(\frac{2 c_{p} d_{31} t_{p}}{a \varepsilon}\right) A_{i n}^{2}}{\left(4 \zeta^{2}+k_{31}^{2}\right)\left(R C_{b} \omega\right)^{2}+\left(4 \zeta k_{31}^{2}\right)\left(R C_{b} \omega\right)+4 \zeta^{2}}
$$

The elasticity constant $c_{p}$, the piezoelectric coefficient $d_{31}$ and the electrical permittivity $\varepsilon$ are material parameters. The damping ratio $\zeta$, the coupling coefficient $k_{31}$, the wiring $a$, the Laplace transform of input vibrations in terms of acceleration $A_{i n}$, and the beam thickness $t_{p}$ are system properties, and are relatively unaffected by in-plane geometric design changes. The optimal resistance of the circuit element $R_{o p t}$, the capacitance across the piezoelectric layer $C_{b}$, the resonant frequency of the device $\omega$, the average strain in the piezoelectric layer $S_{a v g}$, and the ratio $k_{2}$ of the
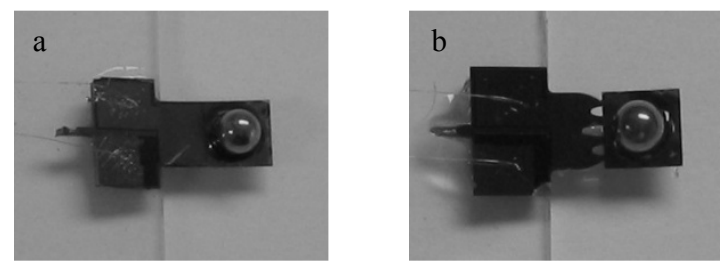

Figure 1: Picture of a) a typical rectangular structure and of b) a structure designed using the BESO material distribution method and a linear finite element model. Dimensions close to those shown in Figure 2 were used for both devices.

Table 1: Material Properties

\begin{tabular}{|l|c|c|}
\hline \multicolumn{1}{|c|}{ Parameter } & Symbol & Value \\
\hline elasticity constant & $c_{p}$ & $170 \mathrm{GPa}$ \\
piezoelectric coefficient & $d_{31}$ & $2 \mathrm{pC} / \mathrm{N}$ \\
electrical permittivity & $\varepsilon$ & $10 \times 8.85 \mathrm{pF} / \mathrm{m}$ \\
\hline
\end{tabular}

Table 2: System Properties

\begin{tabular}{|l|c|c|}
\hline \multicolumn{1}{|c|}{ Parameter } & Symbol & Value \\
\hline damping ratio & $\zeta$ & .02 \\
coupling coefficient & $k_{31}$ & .1221 \\
wiring & $a$ & 1 \\
acceleration & $A_{\text {in }}$ & $4.905 \mathrm{~m} / \mathrm{s}^{2}$ \\
beam thickness & $t_{p}$ & $20 \mu \mathrm{m}$ \\
\hline
\end{tabular}

Table 3: System Parameters

\begin{tabular}{|l|c|}
\hline \multicolumn{1}{|c|}{ Parameter } & Symbol \\
\hline optimal circuit element resistance & $R_{\text {opt }}$ \\
capacitance across piezoelectric layer & $C_{b}$ \\
resonant frequency & $\omega$ \\
average strain in piezoelectric layer & $S_{\text {avg }}$ \\
ratio of beam's end displacement to $\mathrm{S}_{\text {avg }}$ & $k_{2}$ \\
beam surface area & $S A$ \\
\hline
\end{tabular}

beam's end displacement to $S_{a v g}$, are parameters that are direct functions of changes in the geometric design. These parameters and their values are summarized in Tables 1,2, and 3 .

The optimal resistance can be calculated from:

$$
R_{o p t}=\frac{2 \zeta}{C_{b} \omega \sqrt{4 \zeta^{2}+k_{31}^{2}}}
$$




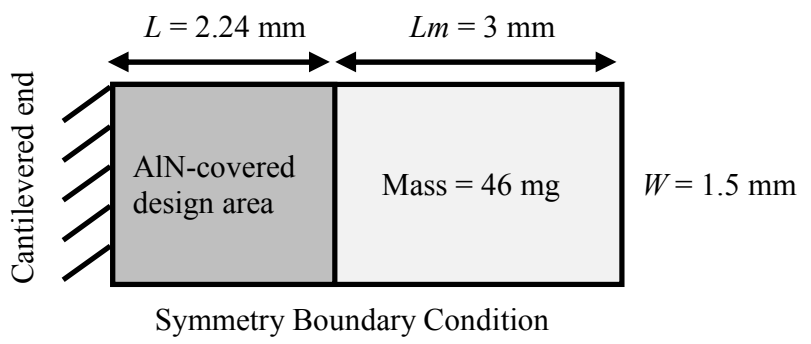

Figure 2: Drawing with dimensions and boundary conditions of sample device used throughout. Thickness of Si beam is $20 \mu \mathrm{m}$, thickness of AlN is $1 \mu \mathrm{m}$. Subject to out-of-plane accelerations.

The capacitance across the piezoelectric layer is:

$C_{b}=\frac{a^{2} \varepsilon^{*} S A}{t_{p}}$

where $S A$ is the surface area of the design area, which is coincident with the area covered by the piezoelectric film.

In S. Roundy (2003) [3], the resonant frequency, the average strain, and the beam's end displacement are calculated using standard beam theory, which does not capture the nonlinear behavior of these devices. Here, these parameters are obtained from a finite element model that will be described in detail in a later section.

\section{Geometry-dependent power output}

In the past, optimizations have been conducted on circuit design, piezoelectric configurations, relative device dimensions, and electromechanical coupling properties [3] [4] for example. The focus here is on mechanical design. In particular, as shown in Fig. 2, the design area consists of the area cantilevered on the left and attached to the mass on the right. The thicknesses of the structural and piezoelectric layers remain unchanged after redesign. From an initial rectangular area, topology design consists of the introduction of holes in the structure, as well as the determination of the shape and dimensions of those holes and of the structure's boundaries.

The material properties are not dependent on topology design, and it is assumed that the system properties have a relatively weak dependence on topology. In addition, if linear beam theory is used as a first approximation for this derivation, the average strain to vertical displacement parameter $k_{2}$ is given by:

$$
k_{2}=\frac{1}{\omega^{2} S_{a v g}} \frac{F_{\text {mass }}}{m}
$$

Where $F_{\text {mass }}$ is the force on the beam due to the end mass, and $m$ the mass itself.

The power output is then roughly proportional to the device frequency, the piezoelectric surface area, and the square of the average strain in the piezoelectric layer:

$$
P \alpha\left(\omega_{n}\right)(S A)\left(S_{\text {avg }}\right)^{2}
$$

Thus, given an operating frequency, and assuming that the effect from increasing the average strain dominates the effect from decreasing the surface area, the problem of increasing the power output is roughly equivalent to that of increasing the average strain in the piezoelectric layer. The maximum stress in that layer should have the same value for all devices being compared, and is set by material strength considerations. An optimal power output is therefore achieved for a uniform stress distribution across the piezoelectric layer.

\section{Geometric Nonlinearities}

Geometric nonlinear behavior is expected from thin plates subjected to large displacements [5]. The in-plane strains cause an out-of plane spring hardening, resulting in nonlinear kinematic relations.

\section{The Material Distribution Algorithm}

The BESO [6] material distribution algorithm has been implemented using the ANSYS parametric design language (APDL). The method distributes material in a design area such that as the algorithm advances, stress is increasingly uniform throughout that area. BESO requires an initial guess structure, which is the simple rectangle in this case. The guess structure is discretized, and its response to an input acceleration is found using the finite element method. In general, the elements within the design area with the lowest strain energies are deactivated, yielding a new structure. The stiffness matrix multiplier for deactivated elements is $10^{-6}$. Deactivated elements contribute no mass to the structure. In later iterations, the response to the same input acceleration is found for the structure obtained in the previous iteration. Elements with the lowest strain energies are deactivated, while previously deactivated elements around those with the highest strain energies are reactivated. In addition, the algorithm has been implemented to prevent checker-boarding, and to enhance convergence based on previous iterations.

\section{RESULTS}

\section{Piezoelectric Energy Scavenger}

The energy scavenger shown in Fig. 1a and Fig. 2 is a cantilevered silicon beam, with an area covered with a $1 \mu \mathrm{m}$ AlN piezoelectric film, and an area covered by a mass. The scavenger is designed to operate around $100 \mathrm{~Hz}$, and dimensions are shown in Fig. 2.

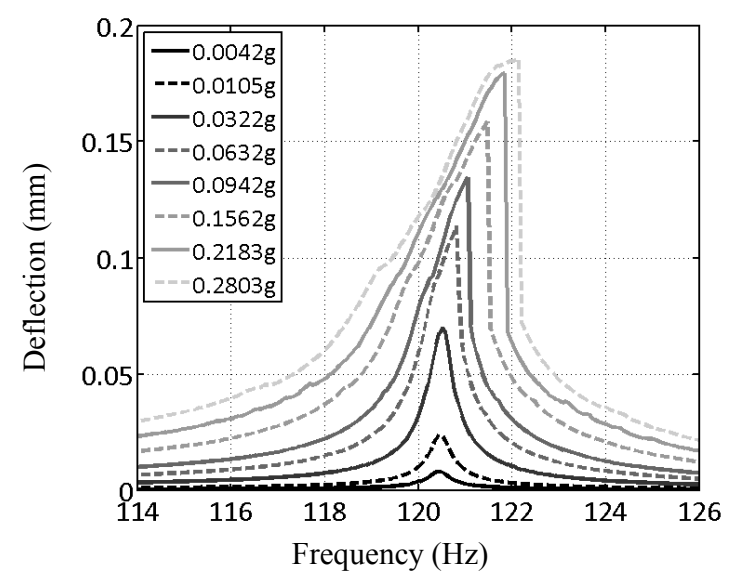

Figure 3: Experimental nonlinear frequency response of a typical device for a range of accelerations. Deflection is measured at center of width, at $x=2.24 \mathrm{~mm}$ using laser Doppler velocimetry $(L D V)$. 


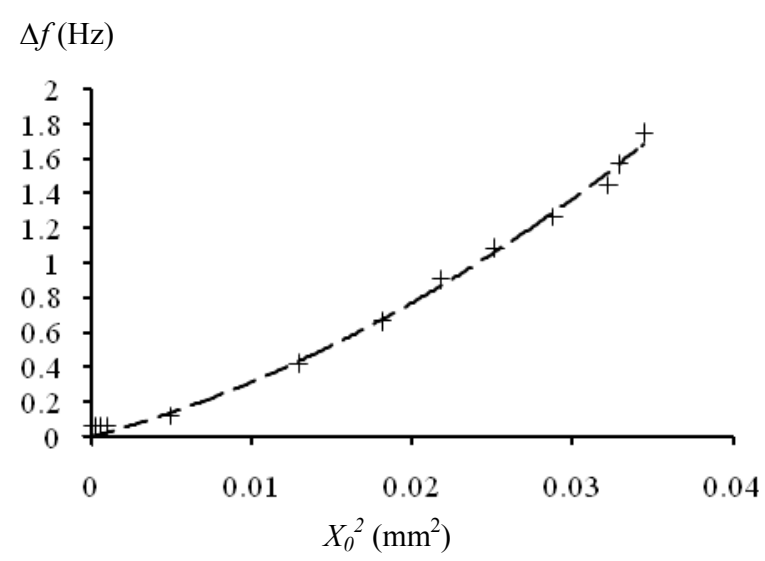

Figure 4: Measured frequency shift plotted against the square of the maximum amplitude at the peak oscillation frequency. The dotted line is the quadratic fit to the experimental data such that $\Delta f=713\left(X_{0}^{2}\right)^{2}+24\left(X_{0}^{2}\right)$, where $\Delta f=\Delta \omega / 2 \pi$, and $X_{0}$ is in millimeters.

\section{Device Nonlinearities}

Figure 3 shows the experimental nonlinear frequency response of a typical device. The spring hardening observed is typical for thin beams subjected to large displacements [5]. These deformations are typical of many vibration-based scavenging devices. Stresses obtained from a linear model are artificially high. For example, if the structure considered in this paper is subjected to $0.5 \mathrm{~g}$ of acceleration, and a quality factor of $Q=250$ is assumed, the linear theory would predict the average stress in the piezoelectric layer to be $23 \%$ higher than the one obtained using a more accurate nonlinear model. In addition, the stress distributions due to linear deformations differ from those created from nonlinear deformations; a nonlinear model should be used for stress-based design.

\section{Modeling nonlinearities}

An attempt was made to model the device nonlinearities using the theory presented by V. Kaajakari [7]. In that theory, it is assumed that the following relationship holds:

$\Delta \omega=\omega_{0}{ }^{\prime}-\omega_{0}=\kappa \omega_{0} X_{0}^{2}$

where $\omega_{0}$ ' is the peak nonlinear oscillation frequency for a given input acceleration, $\omega_{0}$ is the linear resonant frequency, $\Delta \omega$ is the peak frequency shift, $\kappa$ is a proportionality factor, and $X_{0}$ is the maximum amplitude at the peak oscillation frequency. However, as shown in Fig. 4, the harvester peak frequency shift varies quadratically with $X_{0}^{2}$, not linearly. This may indicate that in addition to typical stress stiffening there may also be a more complex quality factor dependence on oscillation amplitude.

Although a complete model of the nonlinear response of the harvester has not yet been achieved, the frequency shift can easily be attributed to a stress stiffening effect. A finite element model of the harvester was implemented using 4-noded shell elements with a $0.05 \mathrm{~mm}$ size, and silicon material properties. As shown in Fig. 2, a clamped boundary condition is applied on the left edge, a symmetry boundary condition on the bottom edge, and mass elements are added in the mass area. The geometric nonlinearities

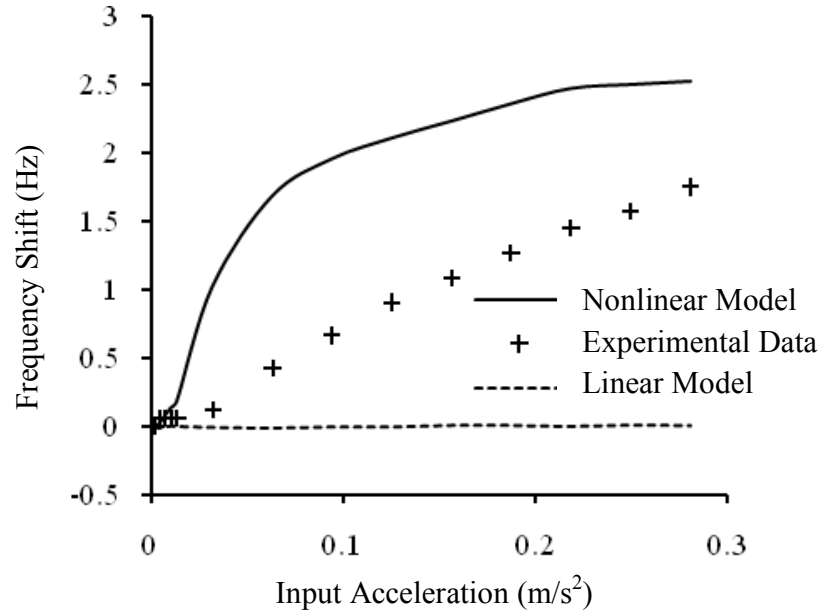

Figure 5: Estimated modeled frequency shifts and experimentally measured frequency shifts plotted against input acceleration. Estimated modeled frequency shifts are based on an equivalent stiffness and on equation 7. The model that includes geometric nonlinearities supplies a frequency shift of the correct order of magnitude but does not model the acceleration dependence well.

are modeled by turning on the NLGEOM option in ANSYS and by applying the load incrementally using approximately 300 substeps.

An equivalent stiffness is defined as the ratio of the input forcing to the maximum static deflection at the in-plane center of the mass. The input forcing is defined as $F=m a Q$ where the mass $m=26.4 \mathrm{mg}$ is an effective mass such that the resonant frequency found from a linear eigenfrequency analysis corresponds to the experimentally measured small displacement frequency. The effective mass compensates for the fabrication uncertainties and for the fact that although the mass is modeled as being distributed over the entire mass area, it is actually a sphere glued to a smaller area. The quality factors were chosen such that both models would predict that the deflection at $L=2.24 \mathrm{~mm}$ be equal to the experimental deflection at that same location. As a result, the models provide information regarding the amount of forcing required to deflect the device by a certain amount; they provide an equivalent stiffness.

A precise peak oscillation frequency cannot be found from an equivalent stiffness since the stiffness is a function of amplitude during the oscillation. However, a rough estimate of the shift in peak oscillation frequency due to the stiffening can be obtained by using the following expression:

$\Delta f=\frac{1}{2 \pi} \frac{1}{\sqrt{m}}\left(\sqrt{k_{1}}-\sqrt{k_{2}}\right)$

where $\Delta f$ is the frequency shift over the acceleration range considered, and $k_{1}$ and $k_{2}$ are the equivalent stiffness values at the beginning and end of the acceleration range respectively. Figure 5 compares the frequency shifts found from the equivalent stiffness values obtained with both the linear and nonlinear finite element models and the experimentally measured frequency shifts. As expected, the frequency shift predicted by the linear model is null since the equivalent stiffness is independent of input forcing, while stiffening is predicted in the nonlinear case. The nonlinear model 


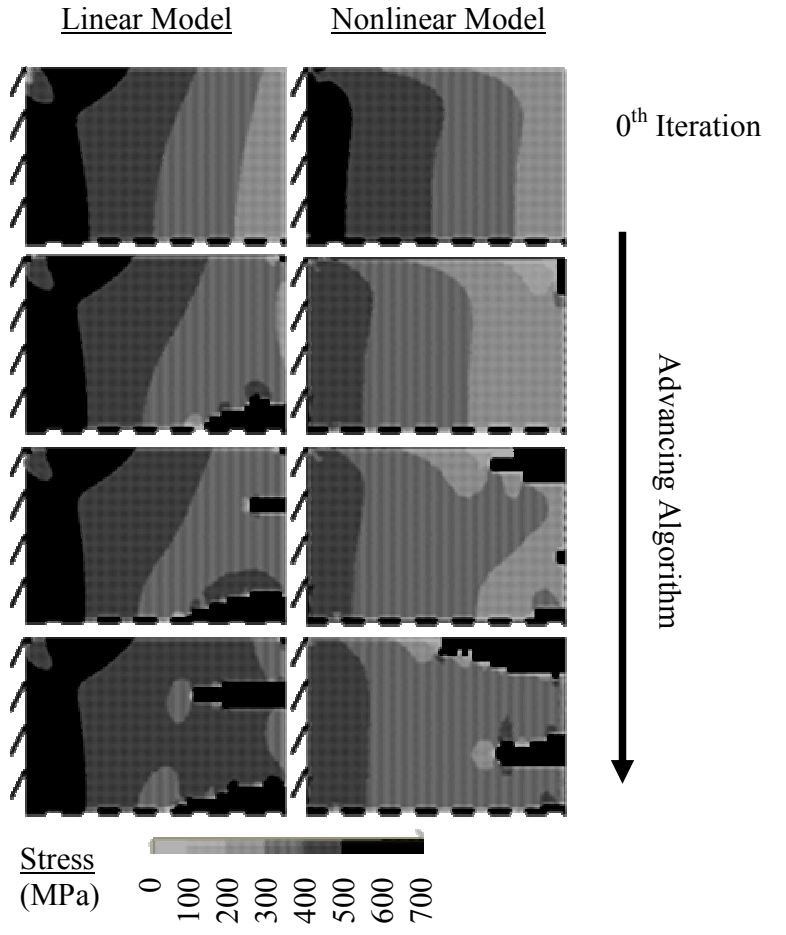

Figure 6: Description of the BESO material distribution algorithm. Stress distribution plots for AlN-covered design areas as the algorithm advances. Results from both linear and nonlinear models are shown. Cantilevered ends on left side and symmetry lines at bottom are shown on each plot. Mass areas are not included. Dark areas are those where material has been removed. Results demonstrate how difference between linear and nonlinear solutions is related to differences in stress distributions.

predicts a maximum frequency shift of $2.5 \mathrm{~Hz}$ at an input acceleration of $0.2803 \mathrm{~m} / \mathrm{s}^{2}$, which is on the same order of magnitude as the experimentally observed $1.7 \mathrm{~Hz}$ shift at that same acceleration. The stress stiffening phenomenon can therefore be considered to be the major cause of the nonlinear behavior observed, although a full model has not yet been achieved.

\section{BESO Material Distribution}

The BESO material distribution algorithm has been implemented for both the linear and nonlinear finite element mechanical models described previously. When nonlinear modeling is used, the resulting structure converges towards a trapezoidal shape with a small central hole. In contrast, if linear modeling is used, the algorithm favors central holes. This is a direct consequence of the differences in stress distributions shown in Fig. 6, and of the logic of the algorithm, removing material from the lowest stressed areas. Preliminary implementations of the BESO algorithm result in a $20 \%$ theoretical increase in power output for an unchanged maximum stress and a frequency that remains within $5 \%$ of the original one.

\section{CONCLUSIONS}

A material distribution algorithm has been applied to a piezoelectric vibrational energy scavenger. By departing from the default rectangular beam structure, and allowing the algorithm to add holes of various shapes and sizes in the structure, a more uniformly distributed stress is obtained in the piezoelectric layer. This results in a device that theoretically has a higher power output than a rectangular structure with an equivalent footprint and maximum stress value.

Geometric nonlinearities have been observed experimentally, and are explained in part by the large deformations being imposed by a large mass on a thin beam. Nonlinear finite element modeling has partially captured this phenomenon. The nonlinearities were shown to have a significant effect on the BESO material distribution algorithm since the stress distribution is different than that in a linear model. Obtaining an accurate nonlinear model will be essential in designing a high-performance mechanical structure.

\section{REFERENCES}

[1] C.M. Jha, M.A. Hopcroft, S.A. Chandorkar, J. Salvia, M. Agarwal, R.N. Candler, R. Melamud, B. Kim, T.W. Kenny, "Thermal Isolation of Encapsulated MEMS Resonators". Journal of Microelectromechanical Systems, Vol. 17, No. 1, (2008).

[2] R. Legtenberg, A.W. Groeneveld, M. Elwenspoek, "Combdrive Actuators for Large Displacements", J. Micromech. MicroEng., 6, pp. 320-329, (1996).

[3] S. Roundy, "A Piezoelectric Vibration Based Generator for Wireless Electronics”, Smart Materials and Structures, 13, pp. 1131-1142 (2003).

[4] S.R. Anton and H.A. Sodano, "A Review of power harvesting using piezoelectric materials (2003-2006)", Smart Materials and Structures, 16, R1-R21, (2007).

[5] P. Malatkar, Nonlinear Vibrations of Cantilever Beams and Plates, PhD thesis, Virginia Polytechnic Institute (2003)

[6] O.M Querin and G.P. Steven, "Evolutionary Structural Optimization (ESO) using a Bidirectional Algorithm”, Eng. Computations. Vol. 15 No. 8, (1998), pp. 1031-1048

[7] V. Kaajakari, T. Mattila, A. Oja, and H. Seppä, "Nonlinear limits for single-crystal silicon microresonators", IEEE Journal of Microelectromechanical Systems, Vol. 13, No. 5, pp. 715-724, (2004)

\section{AKNOWLEDGEMENTS}

This material is based upon work supported under a National Science Foundation Graduate Research Fellowship. The project was also funded by Audi, a premium brand of Volkswagen Group and by the Electronics Research Laboratory, the R\&D division of Volkswagen Group of America.

\section{CONTACT INFORMATION}

*M.A. Philippine, tel: +1-650-823-3811; mandyp@stanford.edu 